Hautarzt 2013 $\cdot 64: 349-353$

DOI 10.1007/s00105-013-2587-0

Online publiziert: 26. April 2013

(c) Springer-Verlag Berlin Heidelberg 2013

G. Hofbauer

Dermatologische Klinik, Universitätsspital Zürich

\title{
Phototherapie und Karzinogenese
}

dierender Ribonukleinsäure (RNS) einen weiteren Beitrag leisten dürften [7, 17, 28].

Die häufigste Form des DNS-Schadens durch UVC, das auf der Erde nach Abfilterung durch die Atmosphäre nur unter künstlichen Bedingungen wie z. B. beim Schweißen vorkommt, und durch UVB stellt die Pyrimidindimerbildung dar. Dabei entstehen Cyclobutanpyrimidindimere (CPD) und Pyrimidinpyrimidonphotoprodukte [(6-4)PP]. (6-4)PP entstehen bereits bei niedrigeren Energieniveaus als CPD. Zusätzlich entstehen durch indirekte Mechanismen auch weitere Photoprodukte wie 8-oxo-deoxyguanosine (8-oxodG). Während die Schäden durch UVB und UVC seit Langem klar erkannt und beschrieben sind, ist dies für UVA weniger eindeutig. In den letzten Jahren zeichnet sich ab, dass durch UVA mittels CPD und 8-oxodG Schäden an der DNS hervorgerufen werden, wobei (6-4) PP eine geringe Rolle zu spielen scheinen. Manche Autoren nehmen daher an, dass Tageslicht vor allem in Form von Cyclobutanpyrimidindimeren (CPD) Schäden in der DNS hinterlässt [4].

\section{DNS-Schäden bei Phototherapie}

Verschiedene Wellenlängen kommen bei der Phototherapie zum Einsatz. Am besten beschrieben sind Effekte für PUVA und UVB in ihrer Wirkung auf die DNS.

UVB und "UVB narrow band“ (UVBnb; synonym mit UVB-Schmalband, UVB $311 \mathrm{~nm}$ ) unterscheiden sich um das 10-Fache in der therapeutisch angewandten Energiedosis. Vergleiche bei bioäquivalenten Dosen, d. h. mit einem 10-fachen Energieunterschied, zeigten keine Unterschiede im DNS-Schaden für diese beiden Wellenlängen [47]. Kürzlich jedoch konnte in einem Mausmodell eine höhere Karzinogenese durch UVBnb gegenüber Breitband-UVB nachgewiesen werden, wobei CPD eng mit erhöhter Krebsentstehung assoziiert waren [22].

UVA1 induziert die Bildung von promutagenen $\mathrm{CPD}$, die sich im Vergleich zu UVB ebenfalls im Bereich des p53Gens, eines wichtigen Tumorsuppressorgens, finden, sich aber von den UVB-induzierten Mutationen unterscheiden. $\mathrm{Zu}$ sätzlich zeigt UVA1 mit Dosisabhängigkeit die Bildung oxidierter Purine und solcher mit Ringöffnung als Zeichen oxidativen Schadens [3]. Nach UVA1-Bestrahlung kommt es auch bei suberythematogenen Dosen zu einer Induktion von p53 und in geringem Maße von $\mathrm{Bcl}-2$ in Keratinozyten, was auf einen Genschaden hinweist [8].

Auf der Ebene von p53-Proteinexpression zeigten sich keine Unterschiede zwischen Hauttumoren von PUVA-Patienten und der allgemeinen Bevölkerung [33]. Spinozelluläre Karzinome von Psoriasispatienten nach PUVA-Behandlung wiesen zahlreiche Mutationen im Bereich des Tumorsuppressorgens p53 auf. Ein Großteil dieser Mutationen fand sich in 5'TpGStellen, die typisch für einen Schaden im Zusammenhang mit Psoralenexposition sind, jedoch fanden sich auch zahlreiche CPD, die auf einen UVB-Schaden hindeuten, sodass bei diesen Tumoren PUVA zusammen mit UVB aus der Umgebung oder früherer therapeutischer Exposition eine Rolle spielt [29]. Zusätzlich zu Mutationen im Bereich von p53 konnten bei spinozellulären Karzinomen von PUVA-Patienten häufige Ha-ras-Mutationen festgestellt werden, die ebenfalls zur 
Karzinogenese beitragen können [21]. In Basalzellkarzinomen von PUVA-Patienten lassen sich Mutationen nachweisen, die sowohl auf einen reinen UV-Schaden durch CPD-Bildung als auch auf Psoralenbeteiligung durch Lokalisation an 5'TpG-Stellen hinweisen, sodass die Autoren annehmen, dass der Hauptschaden an der Erbsubstanz durch UVB aus der Umgebung oder Phototherapie stammt und der Anteil des PUVA-bedingten Schadens niedriger anzusetzen sei [35].

Bei den bei der Phototherapie eingesetzten Wellenlängen lassen sich Schädigungen der DNS nachweisen.

Bei Hauttumoren von Patienten, die eine Phototherapie erhalten hatten, lässt sich jedoch der Einfluss der Phototherapie nur schwer ausschließlich der Behandlung zuordnen, da oft ein Mischbild von verschiedenen therapeutischen Wellenlängen und Lichtschaden aus der Umgebung vorlag.

\section{Klinische Karzinogenese nach Phototherapie}

Psoriatiker mit PUVA-Behandlung stellen die bestdokumentierte Patientengruppe mit langfristiger Nachbeobachtung dar. Vor allem das spinozelluläre Karzinom tritt nach PUVA häufiger auf, nicht jedoch das Basalzellkarzinom $[1,11,20,24,34,41$, 45]. Wichtig ist, dass eine Dosisabhängigkeit zwischen PUVA und nachfolgender Krebsentstehung besteht [1,27, 41]. Bei bis zu 150 Sitzungen PUVA-Therapie ließ sich kein Anstieg von Hautkrebsfällen zeigen [41]. Daraus leiten sich die Handlungsempfehlungen $a b$, die auf europäischer Ebene z. B. eine Obergrenze von 250 bis 300 Phototherapiesitzungen auf Lebenszeit empfehlen, um den deutlichen Anstieg an Hautkrebsfällen bei Überschreiten dieser Grenze zu vermeiden [31].

\section{》) Eine Obergrenze von 250 bis 300 Phototherapiesitzungen auf Lebenszeit wird empfohlen}

Die berichtete Häufung von spinozellulärem Karzinom und die grenzwertige $\mathrm{Zu}$ -
Hautarzt 2013 • 64:349-353 DOI 10.1007/s00105-013-2587-0

๑) Springer-Verlag Berlin Heidelberg 2013

\section{G. Hofbauer \\ Phototherapie und Karzinogenese}

\section{Zusammenfassung}

Phototherapie nutzt mit Erfolg die kurzfristige antiproliferative und antientzündliche Wirkung von ultraviolettem Licht. Wegen seiner längerfristigen Wirkung jedoch ist ultraviolettes Licht als Karzinogen anerkannt. Die Wellenlängen der Phototherapie zeigen pathomechanistisch verschiedene karzinogene Wirkungen, zuvorderst Mutationen der Keratinozyten-DNS. Klinisch zeigt PUVA besonders nach 350 Sitzungen auf Lebenszeit ein deutlich erhöhtes Risiko für spinozelluläre Karzinome der Haut. Melanom und genitaler Hautkrebs jedoch sind nicht durch alleinige PUVA erhöht. Vorbestehende UV-Belastung, Immunsuppression und weitere systemische Behandlung erhöhen die Karzinogenese durch PUVA. UVB-Breitband, -Schmalband

(„narrow band") und UVA1 zeigen bisher keine klinische Erhöhung der Karzinogenese, müssen aber in ihren Spätfolgen weiter überwacht werden. Phototherapie ist weiterhin eine sichere Behandlungsmodalität, sofern die Indikation gut begründet ist, Vorbehandlungen sowie Begleitmedikation und -umstände berücksichtigt, eher kurze und dosisintensive Protokolle gewählt werden, PUVA als Zweitlinienbehandlung eingesetzt wird und 250 bis 300 Sitzungen Phototherapie auf Lebenszeit nicht überschritten werden.

Schlüsselwörter

Weißer Hautkrebs · Basalzellkarzinom .

Spinozelluläres Karzinom · Melanom . UV-Licht

\section{Phototherapy and carcinogenesis}

\section{Abstract}

Phototherapy successfully uses the shortterm effects of ultraviolet light against inflammation and proliferation. For its longterm effects, however, ultraviolet light was recently classified as a carcinogen. The wave spectrum employed in phototherapy has various carcinogenic effects in experimental systems, most notably DNA mutations in keratinocytes. Clinically, PUVA increases the risk for squamous cell carcinoma of the skin, especially after following 350 or more phototherapy sessions over a lifetime. Melanoma and genital skin cancer are not increased by PUVA alone. Previous UV damage, immunosuppression and other systemic treatments increase cutaneous carcinogenesis through PUVA. In contrast, broad-band UVB, narrow-band UVB and UVA1 have not yet been linked to cutaneous carcinogenesis, but will need careful follow-up in larger studies. Phototherapy remains a safe treatment modality, provided that the indication is well-founded, previous exposure and co-carcinogens are considered, and short and dose-intensive treatment protocols are favored, PUVA is chosen as secondline treatment that should not be used for more than a lifetime total of 250-300 phototherapy sessions.

\section{Keywords}

Non-melanoma skin cancer · Basal cell carcinoma - Squamous cell carcinoma . Melanoma · UV light nahme von Basalzellkarzinomen unterscheiden sich zwischen Europa und den USA [1]. Diese Unterschiede dürften in den unterschiedlichen Behandlungsschemata zu suchen sein, wobei in Europa kürzere, aber dosisintensivere Schemata zur Anwendung kamen, während in den USA oft PUVA über längere Zeit mit niedriger Dosis und mit Erhaltungstherapie praktiziert wurde $[1,25,46]$.

Ebenso unterscheiden sich die Berichte für Melanomentstehung zwischen den Kontinenten. Während PUVA-Lentigines häufig gesehen werden, ist die Entstehung von Melanomen nur sporadisch be- richtet worden. Die Ausnahme bildet hier wiederum die Kohortenstudie von Stern et al., in der 15 Jahre nach PUVA ein Anstieg von Melanomfällen verzeichnet wurde. Auch hier treffen jedoch mehrere Risikofaktoren wie systemische Behandlungen der Psoriasis und andere Phototherapien zusammen, sodass PUVA-Behandlung alleine nicht als klarer Risikofaktor für Melanomentstehung belegt werden kann $[1,40,44]$.

Klar erscheint, dass zusätzliche UVBBehandlungen das Risiko für Hautkrebs nach PUVA deutlich erhöhen [20, 23, 
43]. Patienten, die unter PUVA ein spinozelluläres Karzinom der Haut entwickeln, sollten wegen eines erhöhten Risikos für Zweittumore engmaschiger kontrolliert werden [20]. Insbesondere Cyclosporin, das als krebsfördernde Substanz aus der Transplantationsmedizin bekannt ist $[15,17,50]$, steigert das Risiko, nach PUVA-Behandlung spinozelluläre Karzinome zu entwickeln, um das 7-Fache und entspricht damit etwa dem Gewicht von 200 PUVA-Sitzungen [26]. Während Bade-PUVA grundsätzlich dieselben Risiken für Hautkrebsentstehung wie die klassische systemische PUVA birgt [37], ließ sich bisher noch keine Häufung von Hautkrebs nach Bade-PUVA verzeichnen $[12,13]$.

Wie oben geschildert, ist bei Phototherapie zumindest für Cyclosporin ein gesteigertes Risiko für spinozelluläre Karzinome beschrieben. Bekannt ist, dass medikamentöse Immunsuppression mit zu- nehmender Dauer und Dosis das Hautkrebsrisiko besonders für spinozelluläre Karzinome der Haut steigert [10, 17, 36]. Gut belegt ist diese Risikosituation für Organtransplantierte, bei denen eine Phototherapie daher relativ kontraindiziert ist [15]. Lichtschaden generell begünstigt eine Feldkanzerisierung der Haut [18]. Phototoxische Systemmedikation kann diese Feldkanzerisierung verstärken und vor allem multiple spinozelluläre Karzinome der Haut bewirken. Am eindrücklichsten lässt sich dies bei Voriconazol, einem Antimykotikum, beobachten, für das bereits nach Exposition von Monaten bis Jahren eine dramatische Zunahme von spinozellulären Karzinomen auf lichtexponierter Haut beschrieben wurde $[5,38]$. Weniger gut beschrieben ist die häufiger verwendete Substanz Azathioprin, die die Lichtempfindlichkeit der Haut für UVA verdoppelt und einen direkten Schaden der DNS durch UVA er- möglicht [30]. Kürzlich konnten wir zeigen, dass nicht nur die Lichtempfindlichkeit der Haut bei Patienten unter Azathioprin steigt, sondern dass UVA auch zu vermehrtem Genschaden der Keratinozyten führt, ein Zustand, der durch einen Wechsel der Medikation messbar gebessert werden konnte [16]. Auch wenn diese Befunde klar ein erhöhtes Risiko für Hautkrebsentwicklung vermuten lassen, gibt es bisher keine Berichte, die Azathioprin mit vermehrter Hautkrebsentstehung direkt in Verbindung bringen. In anderem Zusammenhang häufig verschriebene Substanzen wie Thiaziddiuretika sind mit einem erhöhten Risiko für epithelialen Hautkrebs verknüpft [19], was wahrscheinlich auf die bekannte Photosensibilisierung durch diese Substanzklasse zurückzuführen ist. Zusammenfassend bergen Systembehandlungen, die in inhaltlichem Zusammenhang mit der durch Phototherapie zu behandelnden Derma-

\section{Hier steht eine Anzeige.}

\section{亚 Springer}


tose oder unzusammenhängend gegeben werden, das Risiko, durch Immunsuppression oder Photosensibilisierung das Risiko für Hautkrebs nach Phototherapie zu steigern. Bei Phototherapie sollte daher immer die gesamte Begleitmedikation genau geprüft und ggf. reduziert oder substituiert werden.

\section{\) Systembehandlungen können Hautkrebs durch vermehrten Lichtschaden steigern}

Besorgnis erregt hatte ein Bericht über $\mathrm{Zu}$ nahme von genitalem Hautkrebs bei lichtbehandelten Patienten [39], wobei diese in der Vorgeschichte auch mit Teerpräparaten behandelt worden waren. PUVA allein konnte jedoch nicht mit genitalem Hautkrebs in Zusammenhang gebracht werden [49]. Auch eine große französische retrospektive Studie konnte einen solchen $\mathrm{Zu}$ sammenhang nicht bestätigen [2], sodass in vielen Lichtabteilungen inzwischen kein Genitalschutz während Phototherapie praktiziert wird.

Grundsätzlich nehmen wir an, dass UVB in der Breitband-, aber auch in der Schmalbandvariante die kutane Karzinogenese steigern müsste [9]. Bisher zumindest ließ sich in Studien kein solcher Anstieg ausmachen [14, 42, 48]. Allerdings ist die langfristige Nachverfolgung von UVB(nb)-behandelten Patienten schwierig [6], sodass wohl nur multizentrische Studien mit jahrzehntelanger Dauer eine Assoziation zwischen UVB und Hautkrebs erkennen lassen werden. Zumindest lässt sich vermuten, dass eine Induktion der kutanen Karzinogenese durch UVB deutlich geringer als die unter PUVA beobachtete ausfällt [48]. UVA1 zeigt auf experimenteller Ebene, wie oben diskutiert, genetische Schäden $[3,8]$, ließ sich aber bisher nicht mit einer klinisch messbaren Zunahme von Hautkrebs in Verbindung bringen.

\section{Fazit für die Praxis}

- Ultraviolettes Licht ist ein Karzinogen für Hautkrebs.
- Phototherapie induziert einen messbaren Genschaden der Haut.

- Spinozelluläre Karzinome der Haut nehmen ab 350 PUVA-Sitzungen auf Lebenszeit stark zu.

- Kurze, dosisintensive PUVA scheint weniger karzinogen als längere, dosisschwache PUVA zu sein.

- PUVA allein ist kein klarer Auslöser für genitalen Hautkrebs oder Melanom.

- UVB-, UVBnb- und UVA1 zeigen bisher keinen messbaren Anstieg von Hautkrebs.

- Die Indikation zur Phototherapie sollte gut begründet sein, Begleitmedikation und Kokarzinogene berücksichtigen, kurze, dosisintensive Protokolle bevorzugen, PUVA möglichst als Zweitlinientherapie einsetzen und 250 bis 300 Sitzungen auf Lebenszeit nicht überschreiten.

- Patienten mit Hautkrebs nach Phototherapie oder mit einer kumulativen Sitzungszahl über $\mathbf{3 5 0}$ sollten engmaschig auf Hautkrebs kontrolliert werden.

\section{Korrespondenzadresse}

Prof. Dr. G. Hofbauer

Dermatologische Klinik,

Universitätsspital Zürich

Gloriastr. 31, 8091 Zürich

Schweiz

hofbauer@usz.ch

Interessenkonflikt. Der korrespondierende Autor gibt an, dass kein Interessenkonflikt besteht.

\section{Literatur}

1. Archier E, Devaux S, Castela E et al (2012) Carcinogenic risks of psoralen UV-A therapy and narrowband UV-B therapy in chronic plaque psoriasis: a systematic literature review. J Eur Acad Dermatol Venereol 26(Suppl 3):22-31

2. Aubin F, Puzenat E, Arveux P et al (2001) Genital squamous cell carcinoma in men treated by photochemotherapy. A cancer registry-based study from 1978 to 1998. Br J Dermatol 144:1204-1206

3. Besaratinia A, Synold TW, Chen HH et al (2005) DNA lesions induced by UV A1 and $B$ radiation in human cells: comparative analyses in the overall genome and in the p53 tumor suppressor gene. Proc Natl Acad Sci U S A 102:10058-10063

4. Besaratinia A, Yoon Jl, Schroeder C et al (2011) Wavelength dependence of ultraviolet radiation-induced DNA damage as determined by laser irradiation suggests that cyclobutane pyrimidine dimers are the principal DNA lesions produced by terrestrial sunlight. FASEB J 25:3079-3091
5. Cowen EW, Nguyen JC, Miller DD et al (2010) Chronic phototoxicity and aggressive squamous cell carcinoma of the skin in children and adults during treatment with voriconazole. J Am Acad Dermatol 62:31-37

6. Diffey BL, Farr PM (2007) The challenge of followup in narrowband ultraviolet B phototherapy. $\mathrm{Br}$ J Dermatol 157:344-349

7. Dziunycz P, lotzova-Weiss G, Eloranta JJ et al (2010) Squamous cell carcinoma of the skin shows a distinct microRNA profile modulated by UV radiation. J Invest Dermatol 130:2686-2689

8. Edstrom DW, Porwit A, Ros AM (2001) Effects on human skin of repetitive ultraviolet-A1 (UVA1) irradiation and visible light. Photodermatol Photoimmunol Photomed 17:66-70

9. El Ghissassi F, Baan R, Straif K et al (2009) A review of human carcinogens - part D: radiation. Lancet Oncol 10:751-752

10. Euvrard S, Morelon E, Rostaing L et al (2012) Sirolimus and secondary skin-cancer prevention in kidney transplantation. N Engl J Med 367:329-339

11. Forman AB, Roenigk HH Jr, Caro WA et al (1989) Long-term follow-up of skin cancer in the PUVA-48 cooperative study. Arch Dermatol 125:515-519

12. Hannuksela-Svahn A, Pukkala E, Koulu L et al (1999) Cancer incidence among Finnish psoriasis patients treated with 8-methoxypsoralen bath PUVA. J Am Acad Dermatol 40:694-696

13. Hannuksela A, Pukkala E, Hannuksela M et al (1996) Cancer incidence among Finnish patients with psoriasis treated with trioxsalen bath PUVA. J Am Acad Dermatol 35:685-689

14. Hearn RM, Kerr AC, Rahim KF et al (2008) Incidence of skin cancers in 3867 patients treated with narrow-band ultraviolet B phototherapy. Br J Dermatol 159:931-935

15. Hofbauer GF, Anliker M, Arnold A et al (2009) Swiss clinical practice guidelines for skin cancer in organ transplant recipients. Swiss Med Wkly 139: 407-415

16. Hofbauer GF, Attard NR, Harwood CA et al (2012) Reversal of UVA skin photosensitivity and DNA damage in kidney transplant recipients by replacing azathioprine. Am J Transplant 12:218-225

17. Hofbauer GF, Bouwes Bavinck JN, Euvrard S (2010) Organ transplantation and skin cancer: basic problems and new perspectives. Exp Dermatol 19:473-482

18. Hu B, Castillo E, Harewood L et al (2012) Multifocal epithelial tumors and field cancerization from loss of mesenchymal CSL signaling. Cell 149: $1207-1220$

19. Jensen AO, Thomsen HF, Engebjerg MC et al (2008) Use of photosensitising diuretics and risk of skin cancer: a population-based case-control study. Br J Cancer 99:1522-1528

20. Katz KA, Marcil I, Stern RS (2002) Incidence and risk factors associated with a second squamous cell carcinoma or basal cell carcinoma in psoralen + ultraviolet a light-treated psoriasis patients. J Invest Dermatol 118:1038-1043

21. Kreimer-Erlacher $H$, Seidl $H$, Back B et al (2001) High mutation frequency at Ha-ras exons 1-4 in squamous cell carcinomas from PUVA-treated psoriasis patients. Photochem Photobiol 74:323-330

22. Kunisada M, Kumimoto $H$, Ishizaki K et al (2007) Narrow-band UVB induces more carcinogenic skin tumors than broad-band UVB through the formation of cyclobutane pyrimidine dimer. J Invest Dermatol 127:2865-2871 
23. Lim JL, Stern RS (2005) High levels of ultraviolet B exposure increase the risk of non-melanoma skin cancer in psoralen and ultraviolet A-treated patients. J Invest Dermatol 124:505-513

24. Lindelof B, Sigurgeirsson B, Tegner E et al (1999) PUVA and cancer risk: the Swedish follow-up s tudy. Br J Dermatol 141:108-112

25. Maier H, Schemper M, Ortel B et al (1996) Skin tumors in photochemotherapy for psoriasis: a single-center follow-up of 496 patients. Dermatology 193:185-191

26. Marcil I, Stern RS (2001) Squamous-cell cancer of the skin in patients given PUVA and ciclosporin: nested cohort crossover study. Lancet 358:10421045

27. McKenna KE, Patterson CC, Handley J et al (1996) Cutaneous neoplasia following PUVA therapy for psoriasis. Br J Dermatol 134:639-642

28. Molho-Pessach V, Lotem M (2007) Ultraviolet radiation and cutaneous carcinogenesis. Curr Probl Dermatol 35:14-27

29. Nataraj AJ, Wolf P, Cerroni L et al (1997) p53 mutation in squamous cell carcinomas from psoriasis patients treated with psoralen + UVA (PUVA). J Invest Dermatol 109:238-243

30. O'Donovan P, Perrett CM, Zhang X et al (2005) Azathioprine and UVA light generate mutagenic oxidative DNA damage. Science 309:1871-1874

31. Paul C, Gallini A, Archier E et al (2012) Evidence-based recommendations on topical treatment and phototherapy of psoriasis: systematic review and expert opinion of a panel of dermatologists. J Eur Acad Dermatol Venereol 26(Suppl 3):1-10

32. Pfeifer GP, Besaratinia A (2012) UV wavelength-dependent DNA damage and human non-melanoma and melanoma skin cancer. Photochem Photobiol Sci 11:90-97

33. Proby CM, Peloux Menage $\mathrm{H}$ du, McGregor JM et al (1993) p53 immunoreactivity in cutaneous PUVA tumors is similar to that in other non-melanoma skin neoplasms. J Cutan Pathol 20:435-441

34. Raiss M, Templier I, Beani JC (2004) Skin cancer and psoralen plus UVA: a retrospective study of $106 \mathrm{pa}-$ tients exposed to a great number of PUVA treatments. Ann Dermatol Venereol 131:437-443

35. Seidl H, Kreimer-Erlacher H, Back B et al (2001) UItraviolet exposure as the main initiator of $\mathrm{p} 53 \mathrm{mu}-$ tations in basal cell carcinomas from psoralen and ultraviolet A-treated patients with psoriasis. J Invest Dermatol 117:365-370

36. Shafaeddin Schreve B, Anliker M, Arnold AW et al (2012) Pre- and posttransplant management of solid organ transplant recipients: risk-adjusted follow-up. Curr Probl Dermatol 43:57-70

37. Shephard SE, Panizzon RG (1999) Carcinogenic risk of bath PUVA in comparison to oral PUVA therapy. Dermatology 199:106-112

38. Singer JP, Boker A, Metchnikoff C et al (2012) High cumulative dose exposure to voriconazole is associated with cutaneous squamous cell carcinoma in lung transplant recipients. J Heart Lung Transplant 31:694-699

39. Stern RS (1990) Genital tumors among men with psoriasis exposed to psoralens and ultraviolet $A$ radiation (PUVA) and ultraviolet $B$ radiation. The Photochemotherapy Follow-up Study. N Engl J Med 322:1093-1097

40. Stern RS (2001) The risk of melanoma in association with long-term exposure to PUVA. J Am Acad Dermatol 44:755-761

41. Stern RS (2012) The risk of squamous cell and basal cell cancer associated with psoralen and ultraviolet A therapy: a 30-year prospective study. J Am Acad Dermatol 66:553-562
42. Stern RS, Laird N (1994) The carcinogenic risk of treatments for severe psoriasis. Photochemotherapy Follow-up Study. Cancer 73:2759-2764

43. Stern RS, Liebman EJ, Vakeva L (1998) Oral psoralen and ultraviolet-A light (PUVA) treatment of psoriasis and persistent risk of nonmelanoma skin cancer. PUVA Follow-up Study. J Natl Cancer Inst 90:1278-1284

44. Stern RS, Nichols KT, Vakeva LH (1997) Malignant melanoma in patients treated for psoriasis with methoxsalen (psoralen) and ultraviolet A radiation (PUVA). The PUVA Follow-up Study. N Engl J Med 336:1041-1045

45. Stern RS, Thibodeau LA, Kleinerman RA et al (1979) Risk of cutaneous carcinoma in patients treated with oral methoxsalen photochemotherapy for psoriasis. N Engl J Med 300:809-813

46. Tanew A, Honigsmann H, Ortel B et al (1986) Nonmelanoma skin tumors in long-term photochemotherapy treatment of psoriasis. An 8-year follow-up study. J Am Acad Dermatol 15:960-965

47. Tzung TY, Runger TM (1998) Assessment of DNA damage induced by broadband and narrowband UVB in cultured lymphoblasts and keratinocytes using the comet assay. Photochem Photobiol 67:647-650

48. Weischer M, Blum A, Eberhard F et al (2004) No evidence for increased skin cancer risk in psoriasis patients treated with broadband or narrowband UVB phototherapy: a first retrospective study. Acta Derm Venereol 84:370-374

49. Wolff K, Honigsmann H (1991) Genital carcinomas in psoriasis patients treated with photochemotherapy. Lancet 337:439

50. Wu X, Nguyen BC, Dziunycz P et al (2010) Opposing roles for calcineurin and ATF3 in squamous skin cancer. Nature 465:368-372

\section{Gründung der Deutschen Gesellschaft für Transitions- medizin e.V.}

Erwachsenwerden ist ein langer Prozess, der für Kinder und Jugendliche nicht selten Probleme mit sich bringt. Das gilt besonders, wenn sie chronisch krank sind. Um bestehende Ansätze und Konzepte zusammenzuführen und weiterzuentwickeln, hat sich jetzt die Deutsche Gesellschaft für Transitionsmedizin e.V. (DGfTM) gegründet. Darin engagieren sich Fachleute mehrerer großer Kliniken und Krankenhausträger Deutschlands. In Deutschland machen Jugendliche mit einem besonderen Bedarf an Gesundheitsversorgung und Gesundheitsförderung in der Altersgruppe aller 14- bis 17-Jährigen einen Anteil von rund $16 \%$ aus. Vielen chronisch kranken Jugendlichen gelingt es nicht, einen so eigenverantwortlichen Umgang mit ihrer Erkrankung zu entwickeln, dass sie den Übergang in die Erwachsenenmedizin ohne Unterstützung umsetzen können. Daher verlieren sie für kürzere oder längere Zeit den Kontakt zur notwendigen Spezialbetreuung. Dies hat teilweise gravierende Folgen für Therapie und Compliance. Die Deutsche Gesellschaft für Transitionsmedizin fördert den fachübergreifenden Austausch aller Disziplinen der Medizin, Psychologie, Sozialmedizin, Genetik und Soziologie mit dem Ziel, gesundheitspolitische Akzeptanz herzustellen und die Behandlung und Begleitangebote kritisch zu überprüfen und zu diskutieren

Quelle: http://transitionsmedizin.de 